\title{
Strategic Alignment of Business Processes
}

\author{
Evan D. Morrison, Aditya K. Ghose, Hoa K. Dam, \\ Kerry G. Hinge, and Konstantin Hoesch-Klohe \\ School of Computer Science and Software Engineering, \\ University of Wollongong \\ \{edm92, aditya, hoa, kgh72, khk789\}@uow.edu.au
}

\begin{abstract}
Strategic alignment is a mechanism by which an organization can visualize the relationship between its business processes and strategies. It enables organizational decision makers to collect meaningful insights based on their current processes. Currently it is difficult to show the sustainability of an organization and to determine an optimal set of processes that are required for realizing strategies. Further, there is not a general framework for strategic alignment that can ease this problem. In this article, we propose such a general framework for strategic alignment, which helps develop a clear understanding of the relationships between strategies and business processes. The framework gives organizations an understanding of the relationship between a set of processes and the realization of a set of strategies; it also shows the optimal set of processes that can achieve these strategies.
\end{abstract}

Keywords: Strategic Alignment, BPM, Strategy Modeling, Requirements Engineering, Governance, Effects, Process Modeling.

\section{Introduction}

Strategic alignment is a method for understanding the nature of a business through the correlation of business processes and strategies. The use of strategic alignment allows an organization to contemplate its longevity and to find how achievable its visions for the future are. Within the realm of service oriented architectures, verification and validation are significant areas of study. Finding correlations between strategies and business processes are a key component of any SOA methodology [2/4818]. In this article, we build on the foundations of model validation for the description of business process alignment to ensure that there is alignment between processes and strategies. The method of alignment discussed in this article will enable organizations to find if they have the right processes to fulfil their strategies; and thus, will form the basis for understanding sustainable businesses. Our framework for alignment follows from the definition of most specification validation problems [8]16] with the extension that we are interested in optimizing the use of processes to fit the given strategies.

During the creation of workflow systems, process designers strive to create process models or designs that can be considered sustainable [2 4|17]. The problem for these activities is in defining the meaning of sustainable process designs 
9 17. There is a need to describe and to be able to explain why a process model is sustainable and necessary in a given setting [89]. By process sustainability, we refer to the long-term effectiveness of a business utilizing efficient processes, measurable through the number of strategies that a business is able to enact. Process models can be viewed as sustainable if they realize part of an organizational strategy. Process models are efficient if when used by an organization they produce optimal results for the organization based on some quality of service (QoS) measure. Organizations are sustainable if all their strategies are realized by a process. The organizational strategy "ensure that employees are happy" and a process designed to make employees happy can be used to illustrate this point. The process would be aligned to the strategy as it realizes the strategy and hence should be considered sustainable. If there are two such processes for making employee's happy, then the optimal process is the process that satisfies a desired QoS description, such as, make employee's happy quickly. By identifying the points of interaction between processes and strategies analysts are able to tell if the processes that they have designed are sustainable.

Results from this work hold numerous benefits for designers who ask What? and How? questions, such as What strategy does this process seek to satisfy? and How is this strategy realized? Through the use of the alignment framework presented in this article, analysts will be able to describe and explain a specific process model's sustainability. The framework that we propose also provides a mechanism to compute the most optimal model of alignment, which shows the best way to realize given strategies in an organization.

The contributions of this article are as follows. First, we propose a framework that grants business analysts the ability to correlate processes with strategies. Secondly, we describe how an organization can find how many of its strategies are realizable by its current processes. Finally, we show how to compute the most optimal set of processes within the organizational process portfolio to satisfy the organizational strategies.

These contributions are discussed in the article in the following order. In 92 we provide a background of the tools and languages that form the basis of this alignment framework. In $\$ 3$, we provide an example scenario that describes a generic human resources department. Then in $\$ 4$ we present the strategic business process alignment framework. Through this work, we have been developing a toolkit that provides automated support of many of the concepts in this framework, which we present in \$5. We compare our work to existing literature in $\$ 6$, then conclude and position our future work in $\$ 7$.

\section{Background}

In this section, we will introduce the set of languages used to describe process models and strategies. In the follow sections, we will use these languages to form a crisp description of strategic business process alignment. 


\subsection{Semantic Process Effects}

A business process model represented in the Business Process Modeling Notation $(\mathrm{BPMN})^{1}$ is a collection of activities, gateways, events, sequence flows, pools, swim lanes, and message flows. Semantic effect annotations [7] offer a means to reason over business process models. By reasoning with process effects, we are able to capture the organizational operation model, i.e., "what does this process do?" This is important as it allows us to understand what happens as a result of a business process execution; and what execution scenarios a process designer has created for the organization. In other approaches that rely on syntactical process analysis, no information as to what processes do can be extracted from the process models. This makes pure syntactic analysis difficult when attempting to answer "what" questions about process models.

Previous work in this area [7] has described a method for semantic annotation of business processes. This is an effective way of adding semantic descriptions to process models as it produces reusable artefacts that can be reasoned over. To construct semantically annotated business process models, analysts annotate activities in the model with descriptions of the changes that occur as a result of the activities execution. Such results are referred to as immediate effects of an activity. For example (see Fig 1), an activity Check employee database for suitable replacement within a human resources process model could have the immediate effect: ConfirmedEligibility. Similarly, the event no suitable replacement found has an immediate effect: $\neg$ HolidayProvisioned. See figure 1 for the process model of this example. We represent each effect as a proposition and consider a set of effects as a sentence constructed by the conjunction of the propositions in the set. We denote a singleton immediate effect with one effect on its own (e.g. $\alpha$ ) and an immediate effect with multiple effects (e.g. $\alpha \wedge \beta$ ) as a set of effects (e.g. $\{\alpha, \beta\})$.

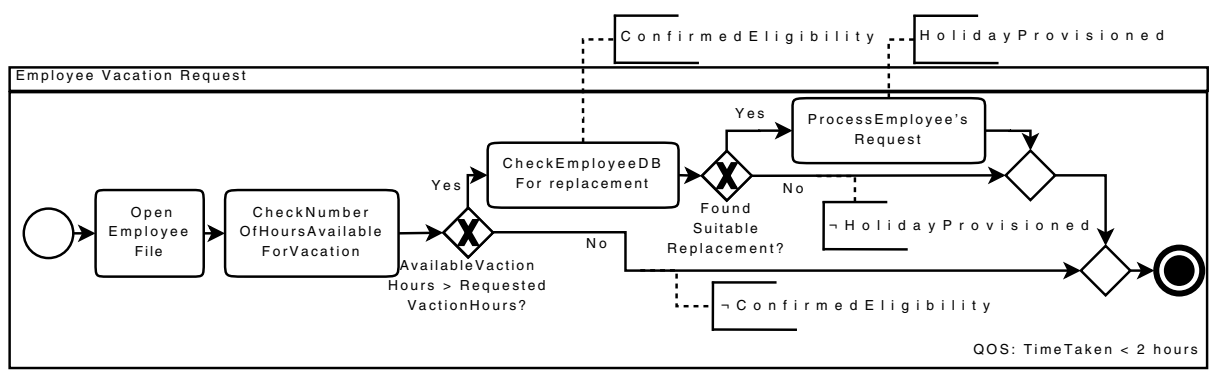

Fig. 1. Employee Vaction Request Process

Each annotation can then be accumulated using a function to produce a semantic description of the process model. Let $e_{a}$ be a set of effects (or a singleton immediate effect) associated with an activity $a$ within a process $P$. Given two

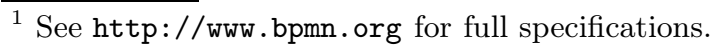


sets of effects $e_{i}$ and $e_{j}$, let a function $\operatorname{acc}\left(e_{i}, e_{j}\right)$ (defined in [7]) return the accumulation of both immediate effects which is a set of possible effect scenarios.

An effect scenario $\epsilon$ is the result of accumulation from a start event $\psi$ in a process to the current activity $a$, where for each pair of sequential activities, the immediate effect of the first activity in sequence is combined with the immediate effect of the next activity in sequence resulting in a cumulative effect for the pair. The cumulative effect of the pair is then accumulated with the immediate effect of the next activity in sequence, and so on until all activities in the sequence have been accumulated over. For each activity there may be multiple effect scenarios, as these show possible execution instances through the process model.

An end effect scenario is an effect scenario associated with an end event in a process, i.e. if $\phi$ is an end event then an effect scenario $\epsilon$ associated with $\phi$ is an end effect scenario. The set of end effect scenarios in a process model is denoted $E_{P}$

A pathway in a process model $P$ from the start event $\psi$ to an activity, event or gateway $a$ is a sequence of activities, events or gateways that can be executed in an unbroken sequence in a manner conformant to the model $P$. We will refer to points in the model $a_{i} \in a_{1}, \ldots, a_{n}$ occuring some point in the model $a_{j}$ before or after other model items in a path.

In this article, we will consider strategic alignment using a notion of process composition. This concept is required for describing business process alignment, as normally, we have found that business process models typically do not realize strategies by themselves, because strategies are described in more general language than process models. For example, a business process that describes a set of activities for evacuating a building will not necessarily satisfy an organizational goal to ensure that employees are safe. In our framework we leverage a composition of processes that contribute to the safety strategy of the organization. There is a general need within businesses to connect similar processes and services that meet the needs and demands of different functional requirements [5]. Processes can be composed using either parallel or sequential process semantics, where the parallel joins have corresponding semantics to a BPMN $A N D$ gateway. A sequential composition has similar semantics to sequential activities within BPMN joined by sequence links.

When discussing process models, we refer to a process portfolio [14] as an organization-wide collection of business process models. Each process in a process portfolio describes the capabilities and activities involved in the execution of each process model. Given our description of process effect accumulation, we will be considering alignment between single processes and strategies, as well as alignment between composed processes and strategies. Given a process portfolio $\mathcal{P}$, we shall use the term composite process portfolio, denoted by $\mathcal{C}_{\mathcal{P}}$, to describe the set of all possible compositions of processes in $\mathcal{P}$.

\subsection{Strategy Modeling Language}

In our previous work [6], we have proposed a language that can be used by senior executives for describing organizational strategies. This language is called 
the strategy modeling language 2 (SML). The core modeling elements of SML are: Functional Goals, Plans, and Optimization Objectives. Goals are general desired outcomes that organizations want to meet and when described in SML, can be evaluted to be in the boolean state of either fulfilled or not fulfilled. For example, the goal Encourage the use of an employee's holiday period, the goal can be evaluated if there is a process that has an effect that results in HolidayProvisioned. Each plan in SML, describes milestones in an organizational strategy. Where the achievement of goals in sequence are key steps that must be completed in a particular order. Plans may follow tactical decisions that describe a plan of progression that will achieve certain higher level goals. For example, a plan "Ensure that staff are the best in the industry may be shown as a sequence of goals": First maintain_high_employee_morale, then, maintain_ongoing_training. An optimization objective in SML is used to discriminate preferences for strategic outcomes. Based on a goal to encourage holiday usage, an optimization object may be minimize wait time for holiday approval; $\min (W T)$. In this next section, we will give an example of strategies that can be expressed in SML.

\section{Motivating Example}

Consider a human resources department. There are three components to this example. First, there is the the strategic landscape (i.e., the strategies the dept. seeks to realize), then a HR Knowledge base and finally a set of business processes.

Strategic Landscape. The strategies of the department, described in SM, are as follows:

- (Goal) Encourage the use of an employee's holiday period HolidayProvisioned

- (Optimization Objective) Minimize wait time for holiday approval $\min (\mathrm{WT})$ (where WT is wait time).

- (Goal) Maintain retention of high-quality staff EmployeeRention $\wedge$ HighlySkilled Worker

- (Plan) Ensure that staff are the best in the industry First maintain_high_employee_morale then, maintain_ongoing_training

1. (Goal) Maintain high employee morale maintain_happy_employee

2. (Goal) Maintain ongoing training maintain_training_provided

HR Knowledge Base. A domain specific knowledge base that describes the HR department. Knowledge base rules are written as a logical consequence (read $A \Rightarrow B$ as material implication).

\section{- HappyEmployee $\wedge$ SalaryPaid $\Rightarrow$ EmployeeRention}

\footnotetext{
${ }^{2}$ The strategy modeling language (SML) has been implemented in a tool and has been used to construct strategy models for both banking organizations and government agencies - see http://www.dlab.uow.edu.au/crc for details of project.
} 
- $\neg$ SalaryPaid $\Rightarrow \neg$ HappyEmployee

- ConfirmedEligibility $\wedge \neg$ HolidayProvisioned $\Rightarrow \neg$ HappyEmployee

- ConfirmedEligibility $\wedge$ HolidayProvisioned $\Rightarrow$ HappyEmployee

- TrainingProvided $\Rightarrow$ HighlySkilledWorker

Business Process Models. Fig 1 and Fig[2 (in the appendix) describe the processes in BPMN with fragments of semantic effect annotation (complete annotation of these processes would be too large to describe in this paper). This example illustrates how even a basic set of effect annotation fragments can deliver considerably desired values. The four processes of interest include an automated vacation request process, a manual vacation request process, a salary payment process and a training process.

\section{Strategic Alignment of Business Processes}

The realization relationship between business process models and goals is critical to strategic alignment analysis and will be defined in this section. We will then expand realization into a relationship that shows alignment between strategies and processes.

\section{Definition 1 (Realization)}

$A$ process $P$ with a set of end effect scenarios $E_{P}$, realizes a goal $G$, if and only if an end effect scenario of $P$ entails $G$, i.e., $\exists \epsilon \in E_{P}$ s.t. $\epsilon \models G$. We will write: $P$ alignedTo $G$ if this is the case.

Consider a set of process models $\left\{P_{1}, \ldots, P_{n}\right\}$ in a process portfolio $\mathcal{P}$. Given a goal $G$, we want to determine if the process portfolio $\mathcal{P}$ is aligned to the goal $G$. Trivially, we have the following basic test: if $\exists P \in \mathcal{P}$ s.t. $P$ alignedTo $G$ then, $\mathcal{P}$ is aligned to $G$; however, we also need to consider the possible compositions of the processes in $\mathcal{P}$. If a goal can be realized by a process in the composite process portfolio then the process portfolio is aligned to the goal.

\section{Definition 2 (Alignment with Goals)}

Let $\mathcal{P}$ be a process portfolio, let $\mathcal{C}_{P}$ be the composite process portfolio derived from $\mathcal{P}$ and let $\mathcal{G}$ be a set of goals. $\mathcal{P}$ is aligned to a single goal $G$ iff $\exists P \in \mathcal{C}_{P}$ s.t. $P$ alignedTo $G$. This is denoted $\mathcal{P}$ alignedTo $G$. We will say $\mathcal{P}$ alignedTo $\mathcal{G}$ iff $\forall G \in \mathcal{G}$. $\mathcal{P}$ alignedTo $G$.

Strategic plans are sequences of strategic goals (or other plans). Each plan describes milestones in an organizational strategy model. Where each goal in the sequence must be achieved before its successor goal. A plan in a strategy model is a temporal sequence of goals.

\section{Definition 3 (Alignment with Plans)}

Let a plan $L$ be a sequence of goals $\left\langle G_{1}, \ldots, G_{n}\right\rangle$. For the plan to be completely realized by a process model (or process models) each pair of consecutive goals $\left\langle G_{i}, G_{j}\right\rangle$ in the plan must be realized. A plan is realized and aligned to a set of processes if all consecutive goal pairs in the plan are realized. Pairs of goals are realizable in the following ways: 
1. Given two processes $P_{k}$ and $P_{l}$, where the processes can be composed in the sequence $\left\langle P_{k}, P_{l}\right\rangle$ to form process $P_{m}$, if $P_{k}$ realizes $G_{i}$ (but not $G_{j}$ ) and $P_{m}$ realizes $G_{i} \wedge G_{j}$ then the process composition $P_{m}$ realizes the goal pair.

2. Given a semantically annotated process model $P_{n}$, where there is an activity a with effect scenario $\epsilon_{a}$ that entails the goal $G_{i}$ and there is an activity $b$ with effect scenario $\epsilon_{b}$, that occurs in the pathway after activity a, that entails $G_{i} \wedge G_{j}$ and there is an end effect scenario of process $P_{n}$ that entails $G_{i} \wedge G_{j}$ then the process $P_{n}$ realizes the goal pair. The effect scenario $\epsilon_{a}$ must not entail $G_{i} \wedge G_{j}$, otherwise the realization order of the goals will be incorrect.

If the above criteria are met and each goal in $L$ is realized in sequence then the process $P$ realizes $L$. This plan based realization can be incorporated in the alignment model shown in Definition 2

To compute optimization objective Alignment, given a strategy $G$, and two processes $P$ and $P^{\prime}$ with alignment relationships $P$ alignedTo $G$ and $P^{\prime}$ alignedTo $G$. We need to add a mechanism for determining which process is a better fit for the strategy. To do this, we refer to a process capability function that computes the value for processes satisfying a particular strategy; similar functions and capabilities are shown in 11. The function will return the best process from a collection of processes that can satisfy the strategy with a given objective function. For example, consider an organizational optimization objective $O$ : 'minimize cycle time' applied to a functional goal encouraging the use of vacation time. Process $P$ may be a manual process that requires the employee to submit leave request forms and find their own replacements, and process $P^{\prime}$ may be an automated process that automatically selects replacement employees and stream lines the approval process. A QoS execution description for process $P$ may be Time $<2$ days, and the QoS execution description for process $P^{\prime}$ may be Time $<2$ hours. Provided that there are no alternative QoS objectives, then the selection function will select process $P^{\prime}$ as being the optimal process to satisfy the goal.

\section{Definition 4 (Alignment with Optimization Objectives)}

Given a strategy $G$, an optimization objective $O$, and two realization scenarios $P$ alignedTo $G$ and $P^{\prime}$ alignedTo $G$, then we refer to the optimal candidate process for realization as the most optimally aligned process P alignedOptimallyToG which is the process that is more preferred based on the optimization objective, i.e. $P \leq_{O} P^{\prime}$ iff $P$, satisfies the optimization objective $O$ better than $P^{\prime}$. Similarly, if for a strategy $G$, there is a set of processes in a process portfolio $\mathcal{P}$ that optimally realizes the strategy, then the realization is denoted $\mathcal{P}$ alignedOptimallyTo $G$.

We observe that this selection of optimal processes has been discussed in other research such as in [15]. Using the previous definitions of goal alignment, plan alignment and optimization objective alignment, we can now tie together an alignment definition that can be used to describe strategic business process alignment.

\section{Definition 5 (Strategic Alignment)}

Let $\mathcal{C}_{\mathcal{P}}$ be the set of the composed of processes of $\mathcal{P}$. Let $\mathcal{G}$ be a collection of strategies. $\mathcal{P}$ alignedOptimallyTo $\mathcal{G}$ iff: 
1. For each $G \in \mathcal{G}:$ (completeness)

(a) $\exists \mathcal{P}^{\prime} \subseteq \mathcal{C}_{\mathcal{P}} . \mathcal{P}^{\prime}$ alignedOptimallyTo $G$

(b) There is no $\mathcal{P}^{\prime \prime} \subset \mathcal{P}^{\prime}$ where $\mathcal{P}^{\prime \prime}$ satisfies condition a. (realization minimality)

(c) $\neg \exists P \in \mathcal{C}_{\mathcal{P}} .(P \wedge G \models \perp)$ (consistency)

2. There is no $\mathcal{P}^{*} \subset \mathcal{P}$ where $\mathcal{C}_{\mathcal{P} *}$ satisfies condition 1. (alignment minimality)

It should be noticable that there are differences in purpose between Definition 5 and Definition 2, as minimality conditions are missing from Definition 2. In this setting we argue that finding the best set of processes that are able to meet an entire organizational strategy is of great importance for both executives and analysts.

We will now step through an example of alignment between an organizations business processes and strategies.

\section{Example 1 (Strategic Alignment Example)}

Recall from the motivating example 3 there are a number of strategic goals to be realized. In the process portfolio, a number of processes are available for analysis to test if they can be utilized in optimal realization of the organizational strategies. For each process there is a QoS metric for TimeTaken annotated at the bottom right of each model.

We must ensure that all strategic goals are realized by the processes in our process portfolio.

First consider the goal: Encourage the use of an employee's holiday period $\rightarrow$ HolidayProvisioned with the optimization objective minimize wait time for holiday approval, to which the Employee Vacation Request is aligned as the effects of this process realize the goal condition and the QoS variable for Employee Vacation Request is minimal compared to the alternative goal realizing process Manual Employee Vacation Request.

Next, we consider the goal: Maintain retention of high-quality staff $\rightarrow$ EmployeeRention $\wedge$ HighlySkilledWorker. From the knowledge base EmployeeRention is achieved if there are processes that ensure HappyEmployee $\wedge$ SalaryPaid.

The Salary Payment Process has the effect SalaryPaid and the Employee Vacation Request process ensures that the effect HappyEmployee is fulfilled. Training Process has the effect HighlySkilled Worker which completes the requirements for the goal to be realized.

Finally, we have a plan with two subgoals: Ensure that staff are the best in the industry. To realize this plan, we construct a composed process where we attempt to satisfy each goal.

For the sub-goal:Maintain ongoing training $\rightarrow$ TrainingProvided the Training Process is aligned as the effects of this process realize the goal condition.

The sub-goal: Maintain high employee morale $\rightarrow$ HappyEmployee can be aligned with Employee Vacation Request as the effects of this process realize the goal condition.

The process composed of the Training Process and the Employee Vacation request realizes the plan. 
On review of this example, we can determine there is no smaller set of processes we could use to realize all the organizational goals from the example case. As a final analysis on this alignment, we can suggest to the organization that it could drop the manual employee vacation request process.

\section{Implementation}

To demonstrate the use of our framework we have sought to extend the functionality of Process Seer through a text based toolkit without BPMN modeler support. Currently the too ${ }^{3}$ (shown in Figure 3 in the appendix) is able to load and test process models for consistency against a rule base. The tool builds sequential and parallel process compositions, then process seer [7] style effect accumulation can be computed on the composed process models to find composition end effect scenarios.

\section{Related Work}

Koliadis et. al. 9] have proposed a framework for aligning business processes to services capabilities. The framework uses semantic effect accumulations over BPMN models to describe relationships mapping effect scenarios to service outcomes. Our framework differs from the framework for alignment in [9] not only through much more detailed and extensible formal descriptions, but also in that we use the strategy modeling language as a basis for goal relations and we also consider ranked realization. The precursor to [9] is described in [8] where Koliadis and Ghose introduce the notion of relating goals (functional goals - from an and/or decomposition tree) with the accumulated effects of processes. This article describes the fundamental relationship between goals and effects showing how processes are related to requirements. Secondly, the article introduces satisfaction goals by the semantic effects of a process. Satisfaction is based on the relationship between process trajectories (or scenario pathways).

Zirpins et. al. 19 have described the alignment of processes to services using capabilities and role based relationships. The work in [19] provides an excellent service adaptation environment that could be leveraged with this work and work in [17] to describe a capability based change management framework.

In the wider spectrum of methods for relating strategic level goals to business processes, Anton [1] has described an approach to process alignment to $e^{3}$ value models through a series of model transformations. The primary focus of Anton's work is on goals and the analysis of what role they play within an organization. In [1], strategy or high level goals are refined to operational goals and are then typed using general goal subdivisions like maintain, achieve, etc. The article introduces a basic notion of constraint satisfaction and process activity ordering for goal plan realization. This work is still in its early phases and does not distinguish between process activities, activity goals, and goal refinements of strategic goals.

${ }^{3}$ For source and further implementation details see http://www.dlab.uow.edu.au/textseer/ 
In [3], Cardoso et. al. have shown a method for eliciting non-functional goals from business processes (with a practical case study in a Brazilian hospital). The authors provide a method for the construction of goal decomposition trees, and then provide a method for composing multiple trees to describe organizational strategy.

For both [13] the work appears to be lacking descriptive details beyond a methodology for constructing candidate models of business/strategy relationships. Neither framework has a method for assessing the correctness of models constructed with their implementations.

A framework for goal operationalism has been rigorously constructed by Leiter, Ponsard et. al. [10]13] showing a crisp goal satisfaction framework that can be used to describe the satisfaction of software systems over time.

Pijpers et. al. have presented a framework and methodology for modeling business strategies called the $e^{3}$ force in [12]. The $e^{3}$ force examines three perspectives of an organization, one of which is focused on business strategy modeling. The other forces in place on an organization are also modeled (these include the value creation perspective and the IT architecture perspective). The use of separation of concerns in Pijper's work aids in clarifying discussions between relevant stakeholders.

Through the literature reviewed, it has become abundantly clear that there needs to be a link between business process models and strategies. The work that is presented in this article provides the next logical and innovative development towards a formalization of the relationships that should exist in any general SOA framework.

\section{Conclusion}

In this article, we have described alignment as a realization relation between a set of process models and a set of strategies. We have presented a formal framework that can be used to show optimal strategic alignment within an organizational context. The framework contains a set of methods for correlating process models to functional goals, strategic plans and optimization objectives. Further, the result of using strategic alignment to determine the alignment between strategies and processes shows an organization the optimal set of processes from a process portfolio that would help them realize their strategies. The results of this work will provide organizational decision makers with a device to understand sustainability in an operational context. The framework that we have presented contributes to a better understanding of strategic business process alignment and further tool support will equipped decision makers with a device to understand sustainability in an operational context. In future work, we will look to extending the definitions of strategic alignment and then show a method for discovering business processes that can meet organizational strategies, attempting to provide a method for rapid business infrastructure development.

Acknowledgements. Work on this project has been supported by the Australian CRC for Smart Services. http://www.smartservicescrc.com.au/, paper tracking number A10155. 


\section{References}

1. Anton, A.: Goal-based requirements analysis. In: International Conference on Requirements Engineering, pp. 136-144 (1996)

2. Bleistein, S.J., Cox, K., Verner, J.: Validating strategic alignment of organizational it requirements using goal modeling and problem diagrams. Journal of Systems and Software 79(3), 362-378 (2006)

3. Cardoso, E.C.S., Almeida, J.P.A., Guizzardi, G., Guizzardi, R.S.S.: Eliciting Goals for Business Process Models with Non-Functional Requirements Catalogues. In: Halpin, T., Krogstie, J., Nurcan, S., Proper, E., Schmidt, R., Soffer, P., Ukor, R. (eds.) BPMDS 2009 and EMMSAD 2009. LNBIP, vol. 29, pp. 33-45. Springer, Heidelberg (2009)

4. Edirisuriya, A., Johannesson, P.: On the Alignment of Business Models and Process Models. In: Ardagna, D., Mecella, M., Yang, J. (eds.) BPM 2008 Workshops. LNBIP, vol. 17, pp. 68-79. Springer, Heidelberg (2009)

5. Feuerlicht, G.: Simple Metric for Assessing Quality of Service Design. In: Maximilien, E.M., Rossi, G., Yuan, S.-T., Ludwig, H., Fantinato, M. (eds.) ICSOC 2010. LNCS, vol. 6568, pp. 133-143. Springer, Heidelberg (2011)

6. Ghose, A.K., Lê, L.S., Hoesch-Klohe, K., Morrison, E.: The Business Service Representation Language: A Preliminary Report. In: Cezon, M., Wolfsthal, Y. (eds.) ServiceWave 2010 Workshops. LNCS, vol. 6569, pp. 145-152. Springer, Heidelberg (2011)

7. Hinge, K., Ghose, A.K., Koliadis, G.: Process seer: A tool for semantic effect annotation of business process models. In: 13th IEEE International EDOC Conference (2009)

8. Koliadis, G., Ghose, A.: Relating Business Process Models to Goal-Oriented Requirements Models in KAOS. In: Hoffmann, A., Kang, B.-h., Richards, D., Tsumoto, S. (eds.) PKAW 2006. LNCS (LNAI), vol. 4303, pp. 25-39. Springer, Heidelberg (2006)

9. Koliadis, G., Ghose, A., Padmanabhuni, S.: Towards an enterprise business process architecture standard. In: IEEE Congress on Services, pp. 239-246 (2008)

10. Letier, E., van Lamsweerde, A.: Deriving operational software specifications from system goals. SIGSOFT Softw. Eng. Notes 27(6), 119-128 (2002)

11. Ortiz, G., Bordbar, B.: Aspect-oriented quality of service for web services: A modeldriven approach. In: ICWS, pp. 559-566 (2009)

12. Pijpers, V., Gordijn, J., Akkermans, H.: Business strategy-it alignment in a multiactor setting: a mobile e-service case. In: Proceedings of the 10th International Conference on Electronic Commerce (2008)

13. Ponsard, C., Massonet, P., Molderez, J., Rifaut, A., Lamsweerde, A., Van, H.: Early verification and validation of mission critical systems. Formal Methods in System Design 30, 233-247 (2007)

14. Rosemann, M.: The service portfolio of a bpm center of excellence. In: Handbook on Business Process Management 2, pp. 267-284 (2010)

15. Vilenica, A., Hamann, K., Lamersdorf, W., Sudeikat, J., Renz, W.: An Extensible Framework for Dynamic Market-Based Service Selection and Business Process Execution. In: Felber, P., Rouvoy, R. (eds.) DAIS 2011. LNCS, vol. 6723, pp. 150-164. Springer, Heidelberg (2011)

16. Wang, H.-L., Ghose, A.K.: On the foundations of strategic alignment. In: Proc. of the 2006 Australia and New Zealand Academy of Management Conference (2006) 
17. Wang, H.L., Ghose, A.K.: Green strategic alignment: Aligning business strategies with sustainability objectives. In: Unhelkar, B. (ed.) Handbook of Research in Green ICT, pp. 29-41 (2010)

18. Zairi, M.: Business process management: a boundaryless approach to modern competitiveness. Business Process Management Journal 3(1), 64-80 (1997)

19. Zirpins, C., Piccinelli, G.: Evolution of service processes by rule based transformation. In: IFIP International Conference on Digital Communities in a Networked Society, pp. 287-305 (2004)

\section{A Appendix}

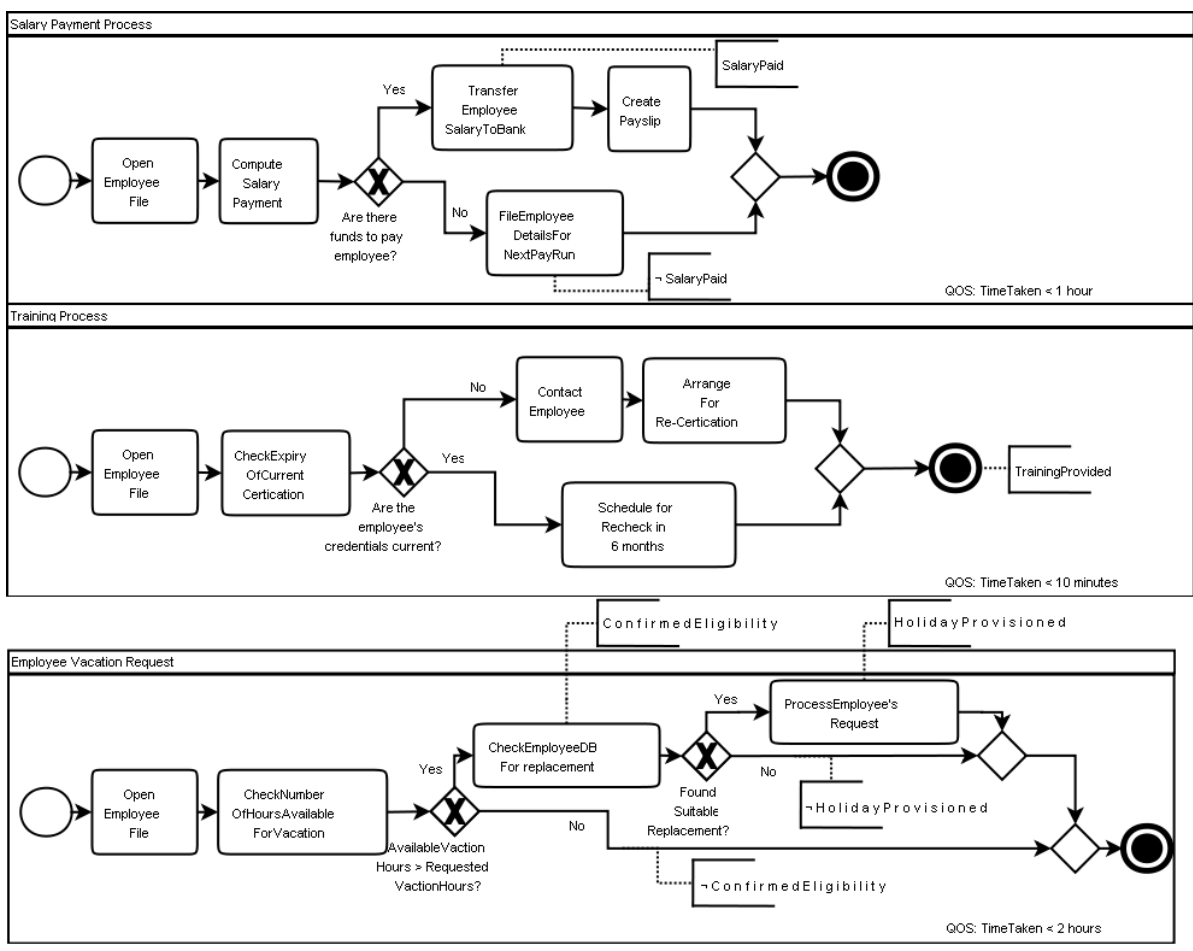

Fig. 2. Process Portfolio Examples : Manual Employee Vaction Request Process, Training Process, Salary Payment Process 


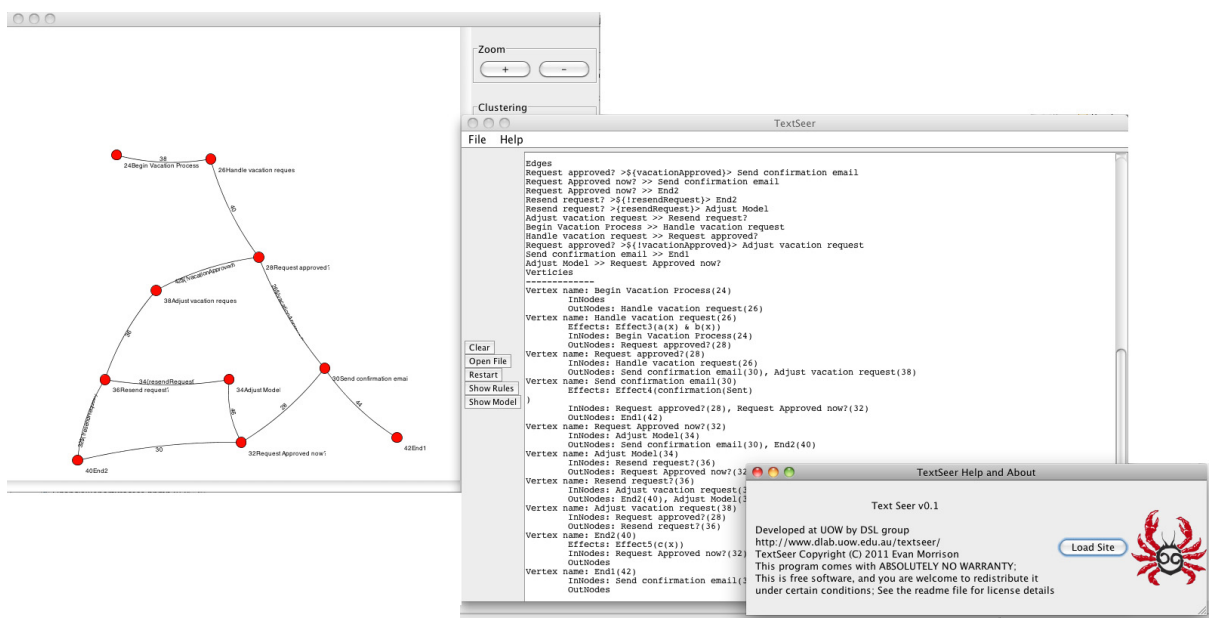

Fig. 3. Screenshot of Toolkita

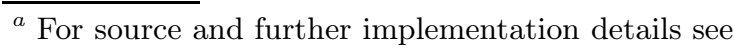
http://www.dlab.uow.edu.au/textseer/ 